\title{
Determining Effective Interface Fracture Properties of 3D Fiber Reinforced Foam Core Sandwich Structures
}

\author{
Zachary T. Kier ${ }^{*}$ and Anthony M. Waas ${ }^{*}$ \\ Composite Structures laboratory, Department of Aerospace Engineering \\ University of Michigan, Ann Arbor, MI.
}

\begin{abstract}
Foam core sandwich composites are finding a wider breadth of use in aerospace, automotive, and construction applications. These structures present unique challenges in terms of material failure and interaction and are highly sensitive to damage and imperfections introduced during manufacturing. An emerging class of 3D Fiber Reinforced Foam Core (3DFRFC) aims to replace monolithic foams used in sandwich structure cores particularly in demanding high performance aerospace applications. This research is focused on investigating the development of testing methods capable of measuring the effective interface fracture properties between the facesheet and the core in 3DFRFCs. Double Cantilever Beam and EndNotched Flexure specimens are developed to evaluate the Mode I and Mode II fracture properties of a 3DFRFC. Preliminary nonlinear finite element analysis with a progressive failure methodology is used to understand the delamination propagation and aid in specimen development. 3DFRFC panels for fracture testing are manufactured and experimental testing will follow final specimen fabrication.
\end{abstract}

\section{Introduction}

Composite sandwich structures provide distinct advantages in aerospace, automotive, and construction industries, affording high specific stiffness compared to metallic components. A particular challenge of utilizing sandwich structures is their sensitivity to manufacturing induced defects, damage, and core-to-facesheet delamination. The ability to assess the residual load carrying capability of sandwich components with such features requires extensive full-scale test programs, detailed and thorough analysis, or likely some combination of the two. The current emphases on cost-reduction tends to shift focus toward less cost-intensive simulation; however, the heavy reliance on simulation and computational analysis requires more careful thought into the coupon level tests that are conducted to acquire the material properties necessary to perform the desired full-scale analysis. This has long been a challenge in determining the fracture properties for composite structures and is further compounded with the addition of bonded cores in sandwich structures. Determining the appropriate method for measuring the facesheet-to-core interface fracture properties of sandwich composites continues to be a challenge as the development of new types of sandwich core materials persist.

\footnotetext{
*NASA Space Technology Research Fellow, Department of Aerospace Engineering, 1320 Beal Avenue, Ann Arbor, MI, 48109, AIAA Student Member, ztkier@umich.edu.

${ }^{\dagger}$ Felix Pawlowski Collegiate Professor of Aerospace Engineering, Department of Aerospace Engineering, 1320 Beal Avenue, Ann Arbor, MI, 48109, AIAA Fellow.
} 
One emerging class of materials has been developed with the potential to affect the ability of sandwich structures to tolerate manufacturing induced defects, damage, and core-to-facesheet delamination. 3D Fiber Reinforced Foam Core (3DFRFC) represents a class of sandwich core materials that consist of low density structural foam reinforced with a three-dimensional, trusslike fiber composite structure that provides added load paths between the facesheets of the composite sandwich and acts to impede crack propagation within the foam. The 3DFRFC architecture can be quite varied through the selection of the reinforcing fiber (glass, carbon, $\operatorname{Kevlar}{ }^{\circledR}$, Spectra ${ }^{\circledR}$, etc.), foam material, foam density, and matrix material, in addition to the overall geometry of the reinforcing truss itself. Some examples of 3DFRFCs include NidaFusion ${ }^{1}$ and TYCOR ${ }^{2}$. A model of a 3DFRFC sandwich structure is shown in Figure 1. The added complexity of 3DFRFC sandwich structures makes the prediction of the global response of full-scale components exceedingly difficult particularly when those structures contain manufacturing defects or damage. The ability to utilize this class of sandwich materials and predict their performance requires an adequate understanding of the constituent interaction and an ability to quantify their damage tolerance.

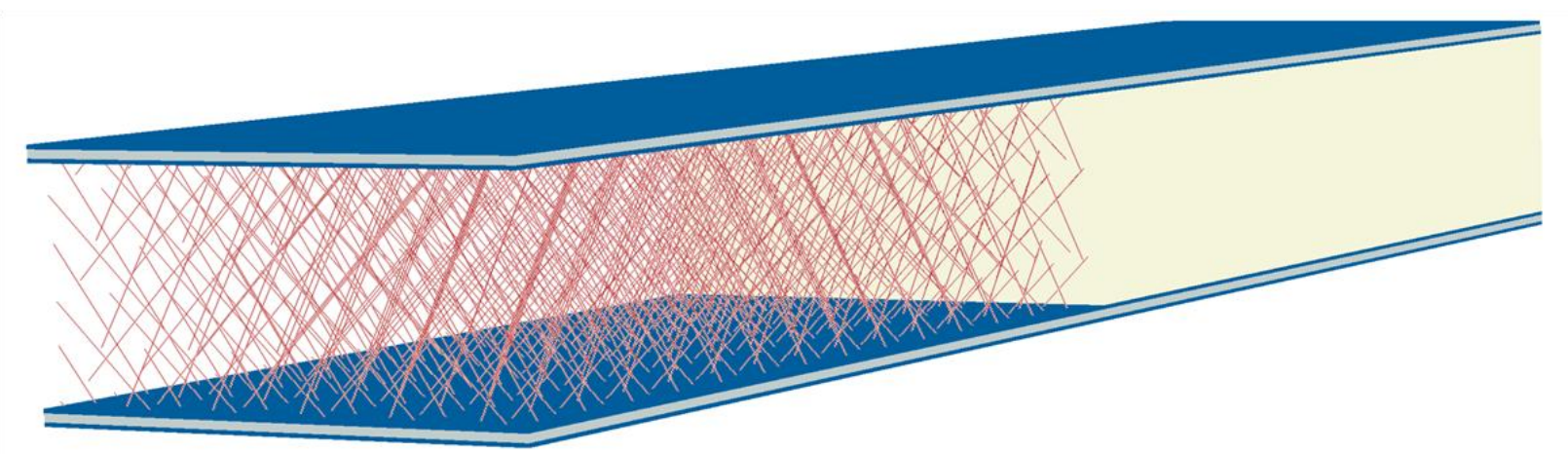

Figure 1. Illustration of a 3DFRFC sandwich composite with region of foam removed. (image not to scale)

This ongoing research aims to develop test methods to experimentally quantify the effective fracture properties of the bonding interface between the core and facesheet in a 3DFRFC sandwich composite. Due to the complexity of the 3DFRFC, a thorough investigation of the test geometry of the test specimens is performed in support of the experimental investigation of the fracture properties of the 3DFRFC sandwich specimens.

\section{Development of 3DFRFC Sandwich Structure Interface Fracture Tests}

The bulk mechanical properties of 3DFRFC sandwich structures are dependent on a variety of factors including the facesheet properties, the foam core properties, the geometry and mechanical properties of the reinforcement within the foam core, and the properties of the adhesive that bonds the facesheets to the core. The interaction of these constituents at the bonding interface is critical to the understanding of the limits of 3DFRFC sandwich structures and is necessary for the development of predictive failure models. The quantification of the fracture properties of the bonding interface is a critical key piece to this process. There has been substantial effort into quantifying the Mode I and Mode II fracture properties of the bonding interface in foam and honeycomb core sandwich structures ${ }^{3-11}$. Many of these approaches are based on various modifications to the Double Cantilever Beam (DCB) test and the End-Notched Flexure (ENF) test to measure the Mode I and Mode II fracture properties, respectively. Many of these methods 
focus on modifications to the loading boundary conditions in an attempt to compensate for the inherent mode mixity that arises from having an offset between neutral axis of the sandwich beam and the intended crack path along the bonding interface. Another approach has been to retain the standard boundary conditions and account for the mode mixity due to the lack of symmetry in the specimen in order to get the relative Mode I and Mode II contributions as is done with the Unsymmetrical Double Cantilever Beam (UDCB) ${ }^{12}$ and the Unsymmetrical End Notch Flexure (UENF) ${ }^{13}$. One key limitation of the UDCB and UENF tests is that they do not allow for direct measurement of the Mode I and Mode II critical energy release rates.

A simpler approach published by Davidson et al. ${ }^{14}$ is used in this study to design test specimens to determine the effective fracture properties of the bonding interface between the core and the facesheet in a 3DFRFC sandwich composite. This is accomplished by designing the specimens so that the neutral axis is coincident with the bonded interface between the facesheet and the core. The specimens are designed such that the neutral axis of the sandwich is collocated with the adhesive interface by bonding an aluminum facing to the facesheet nearest the interface to be tested. Illustrations of the resulting DCB and ENF samples can be seen in Figure 2 and Figure 3 where the initial delamination is shown on the right of the samples at the interface between the upper composite facesheet and the 3DFRFC.

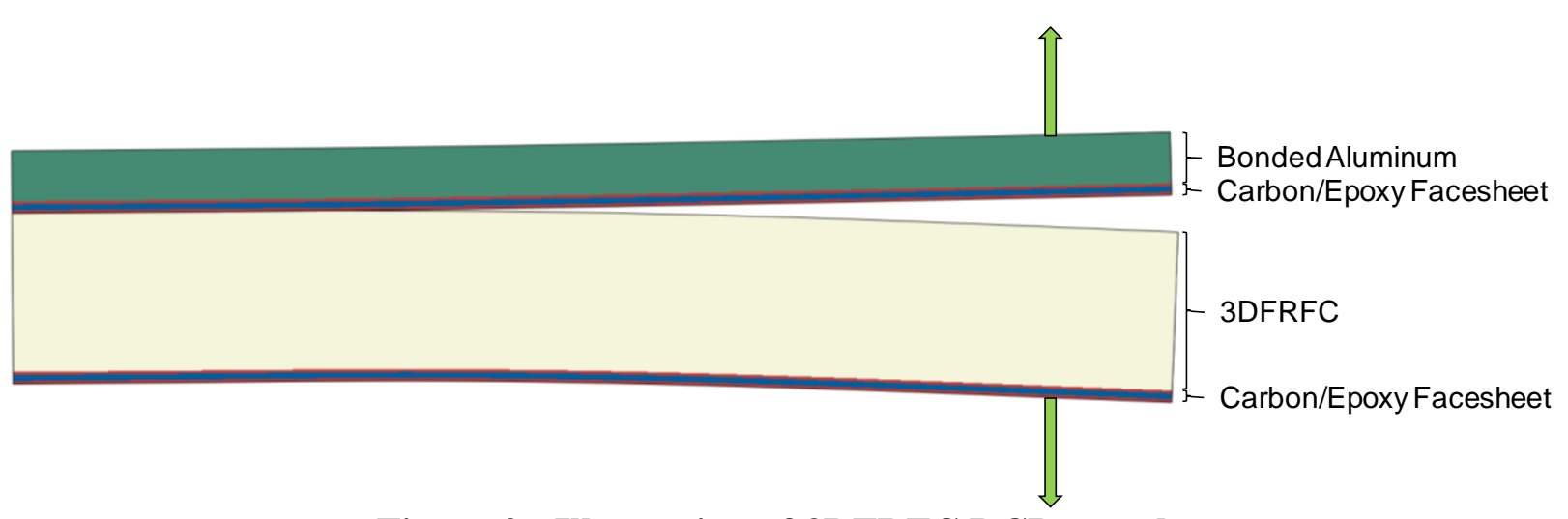

Figure 2: Illustration of 3DFRFC DCB sample.

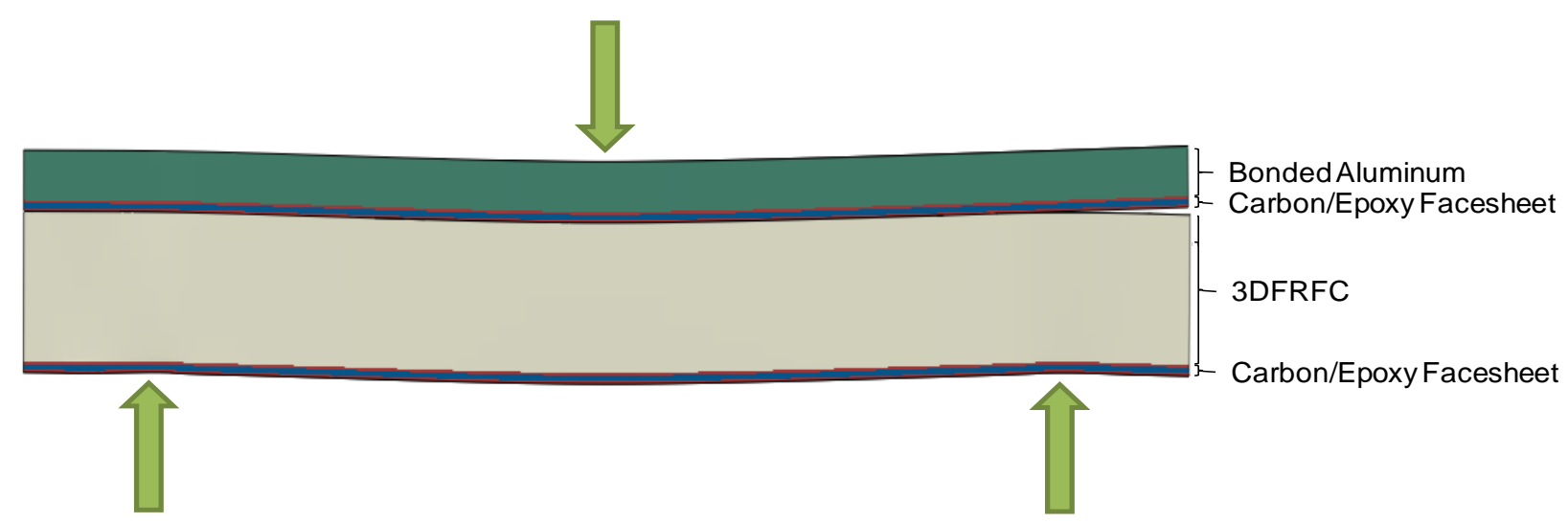

Figure 3: Illustration of 3DFRFC ENF sample.

The bonded DCB and ENF samples were analyzed in order to determine the validity of the experimental method once applied to sandwich composites taking into account the highly orthotropic core properties unique to the 3DFRFC. This was accomplished using finite element 
analysis using the commercial finite element software ABAQUS. The facesheet plies were individually modeled with brick elements and the adhesive layer between the facesheet and the 3DFRFC is modeled with decohesion elements. The initial strength and fracture properties for the interface were based on measured properties for a foam core with equivalent bulk density to the 3DFRFC as an approximate lower bound ${ }^{15}$. The facesheet properties were measured through testing at The Aerospace Corporation ${ }^{16}$, while the mechanical properties of the film adhesive were obtained from vendor data ${ }^{17}$. The effective orthotropic core properties were determined utilizing a micromechanics model recently developed for metallic and pin reinforced foams ${ }^{18}$ that model the reinforcements within the foam as beams on an elastic foundation to derive the effective orthotropic elastic properties for the 3DFRFC based on its specific microstructure and material composition.

One challenge of applying methods developed for foam core sandwich structures to the 3DFRFC is that decohesive zones yield accurate results when the crack plane is well defined, ${ }^{19-21}$ but lose fidelity as the material becomes more discretized and the crack path can no longer be inferred a priori. The added paths for load transfer in the 3D fiber reinforced foam core act to impede crack propagation within the foam and the use of decohesive elements to model this material will likely become less accurate and unable to capture the highly discretized nature of the 3DFRFC particularly in situations with more complex loading. In the current study, the discrete cohesive zone model (DCZM) pioneered by Xie and Waas ${ }^{21}$ is used for modeling delamination between the 3DFRFC and the facesheets due to its increased modeling efficiency.

Finite element analysis of the Mode I modified double cantilever beam specimen using the homogenized orthotropic 3DFRFC material properties resulted in stable crack propagation in Mode I, as intended, Figure 4. While this first step analysis does not account for any additional effects as a result of the discrete nature of the core reinforcement it does illustrate that the global specimen design has the capability to create the conditions for the desired Mode I propagation and is a viable candidate for preliminary testing and further analysis.

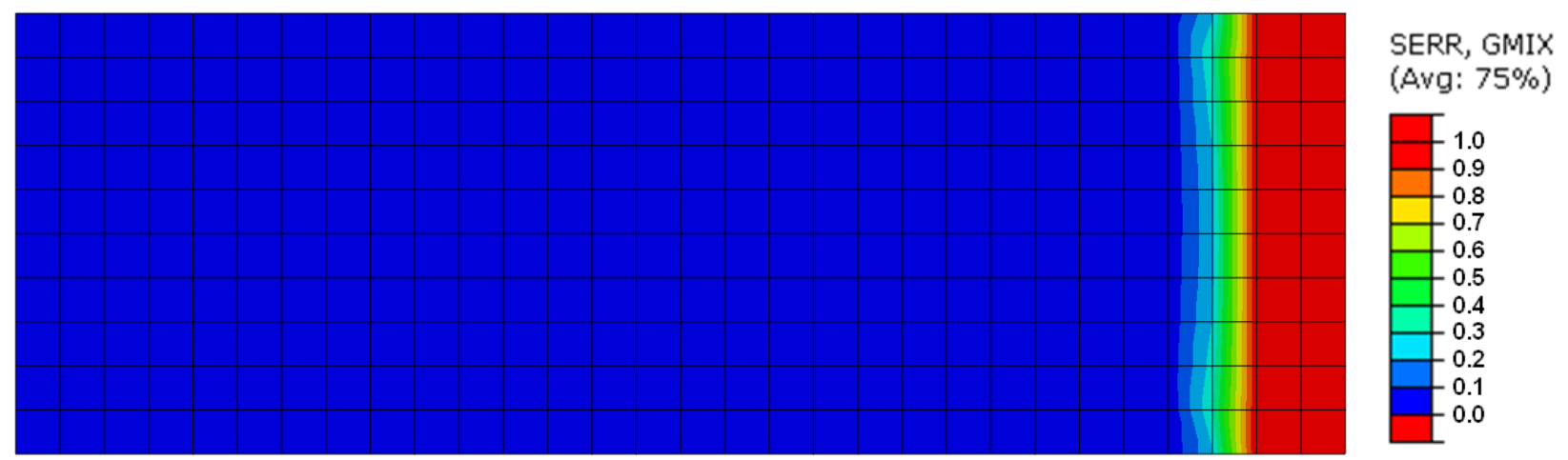

Figure 4: Illustration of fracture surface in DCB model.

Initial results for the Mode II ENF specimen highlighted some additional challenges. Unlike the DCB test, the analysis of the ENF specimen did not yield crack propagation in the desired shear mode. The behavior of the 3DFRFC near the crack front was more complex in the ENF model, resulting in a large region failing in Mode I near the center of the specimen ahead of the initial crack, Figure 5. Additional analysis was conducted to verify whether this phenomenon was a result of the unique orthotropic properties of the 3DFRFC or if the basic specimen design or modeling parameters were faulty. Additional analyses were conducted by replacing the 
orthotropic 3DFRFC core properties in the ENF model with an equivalent isotropic core having the same effective in-plane stiffness as the 3DFRFC. This resulted in crack propagation with the failure occurring primarily in Mode II, as intended, thus exposing the interaction of the highly orthotropic properties of the 3DFRFC as the underlying cause for the change in failure mode from design. The unique interaction of the 3DFRFC material near the crack tip is most clearly highlighted by a comparison of the lateral deformation of the ENF model with the full equivalent (homogenized) orthotropic properties to that of the ENF model with an equivalent isotropic core at the same load point deflection, Figure 6. This comparison shows that the isotropic core exhibits a small amount of lateral expansion near the crack tip (yellow) whereas the model with the full orthotropic properties demonstrates a significant amount of lateral contraction (blue). This structural response is a direct result of the inherent truss structure of the 3DFRFC and results in the localized Mode I behavior of the material despite the global loading conditions. As a result of these findings, additional analysis is being conducted to investigate other loading methods as well as the effect of material orientation on the local material response.
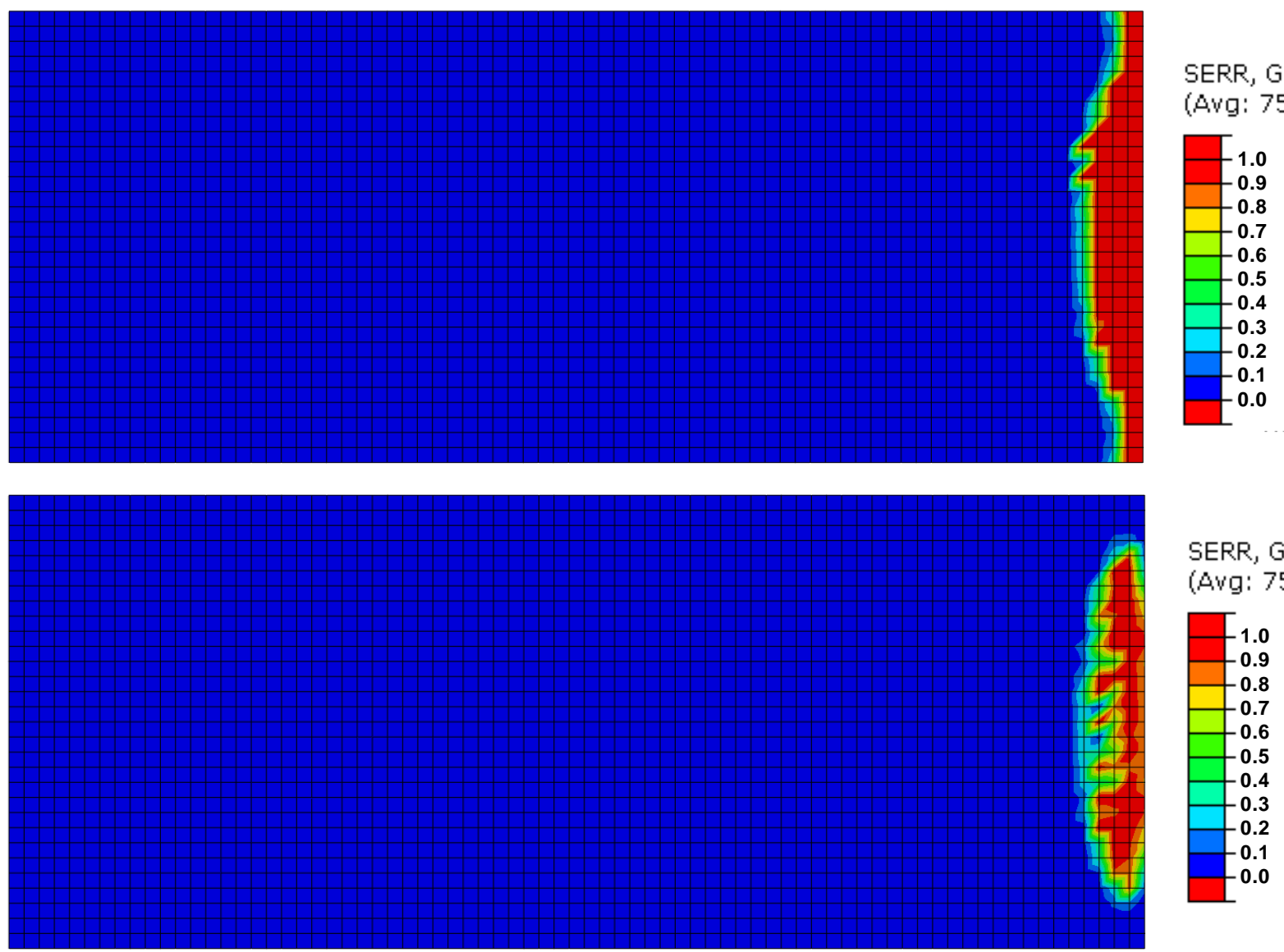

Figure 5: Illustration of fracture surface of ENF model with full orthotropic 3DFRFC properties, top, and area failing in Mode I, bottom. 


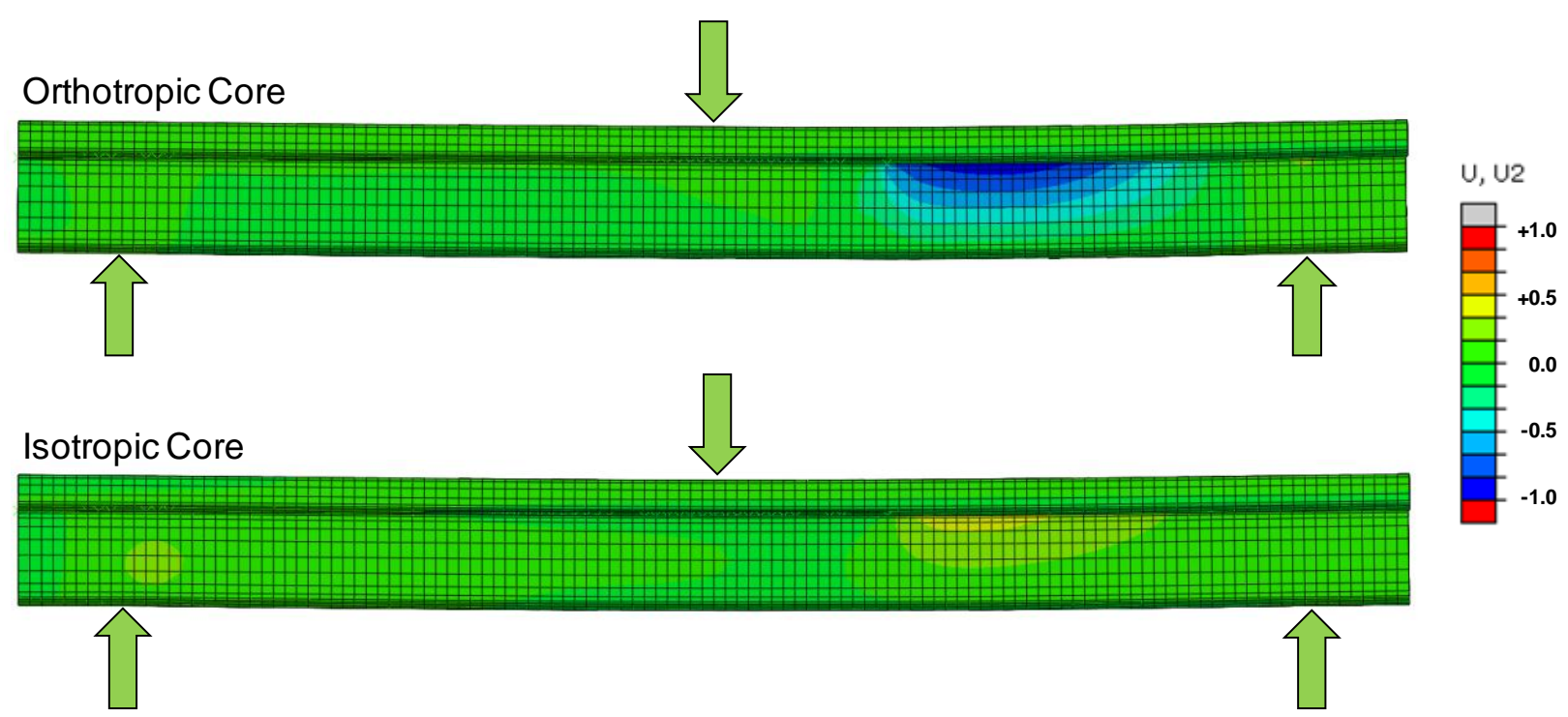

Figure 6: Normalized lateral displacement of ENF models with fully orthotropic 3DFRFC properties and equivalent isotropic properties at the same load-point displacement.

Several possible solutions to alleviate the Mode I contribution in the Mode II tests have been considered including changes in material orientation in the ENF configuration or changing the global boundary conditions to introduce the shear loading through a different loading geometry such as that used in the end-loaded split (ELS) test $^{22}$. A simpler solution was investigated by retaining the 3-point loading configuration but flipping the ENF sample such that the central loading point is contacting the facesheet opposite of the fracture surface and the side supports are contacting the aluminum facing, Figure 7. Initial finite element analysis of this flipped ENF configuration has shown the desired Mode II fracture propagation with essentially no Mode I contribution, Figure 8. This result illustrates that the flipped ENF specimen design has the capability to create the conditions for the desired Mode II propagation and is a viable candidate for preliminary testing and further analysis. Additional analysis is needed to investigate the sensitivity of the configuration to the interface fracture parameters. This configuration does present additional challenges due to the central loading point being located directly on the facesheet. This will have to be addressed in order to insure that localized core crushing and/or facesheet wrinkling does not affect experimental work based on the flipped ENF configuration.

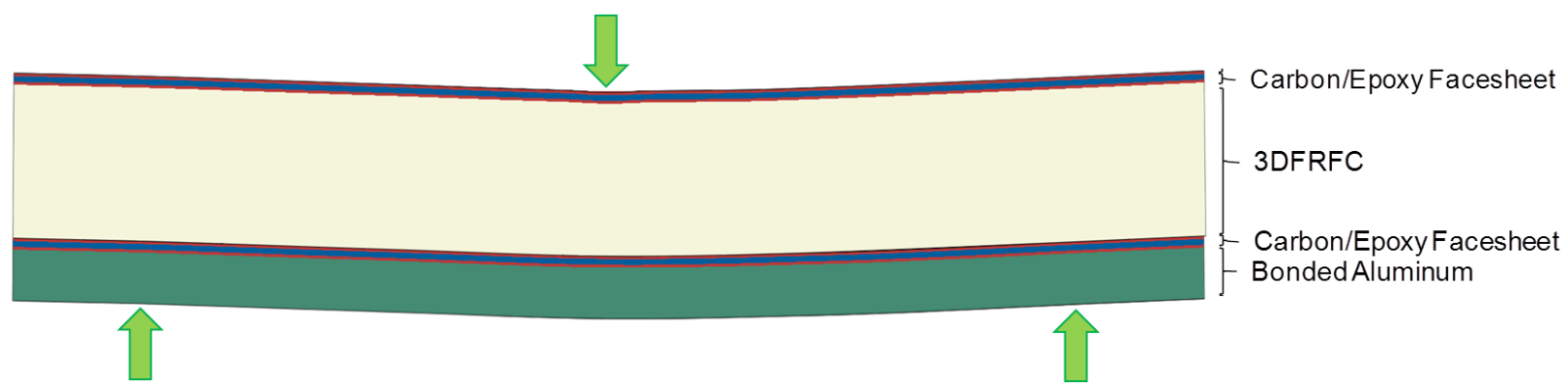

Figure 7: Illustration of 3DFRFC Flipped End-Notched Flexure Sample. 

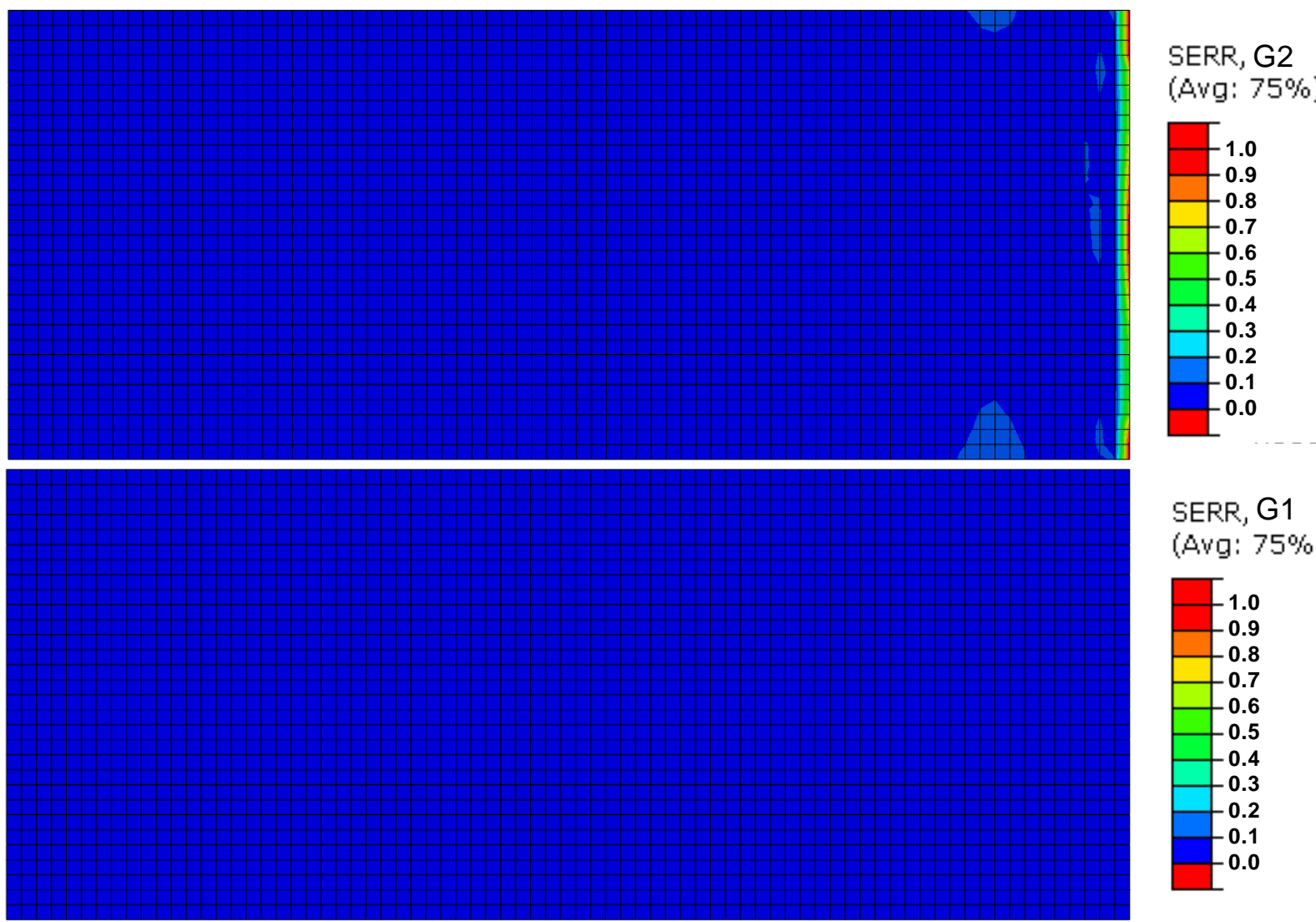

Figure 8: Illustration of fracture surface of flipped ENF model with full orthotropic 3DFRFC properties, top, and Mode I, bottom.

\section{Manufacture of 3DFRFC Interface Fracture Samples}

The general manufacturing procedure for the 3DFRFC interface fracture samples is similar to the one discussed previously for the manufacture of edgewise compression samples with defects $^{23}$ but is included here for completeness. The material system chosen for this investigation is IM7/8552 carbon epoxy for the facesheets and a 0.75 inch thick $12 \mathrm{lb} / \mathrm{ft}^{3} 3 \mathrm{DFRFC}$ for the core. FM® 300 film adhesive is used to bond the facesheets to the core. The desired debonds are manufactured by removing a region of adhesive and replacing it with a PTFE (Teflon®) insert. The panels are inspected via NDE to ensure panel quality and to verify debond placement in the cured sandwich panel prior to removing the desired samples from the fabricated panels. The manufactured 3DFRFC sandwich panels are then cut into samples for aluminum bonding prior to fracture testing, Figure 9. 


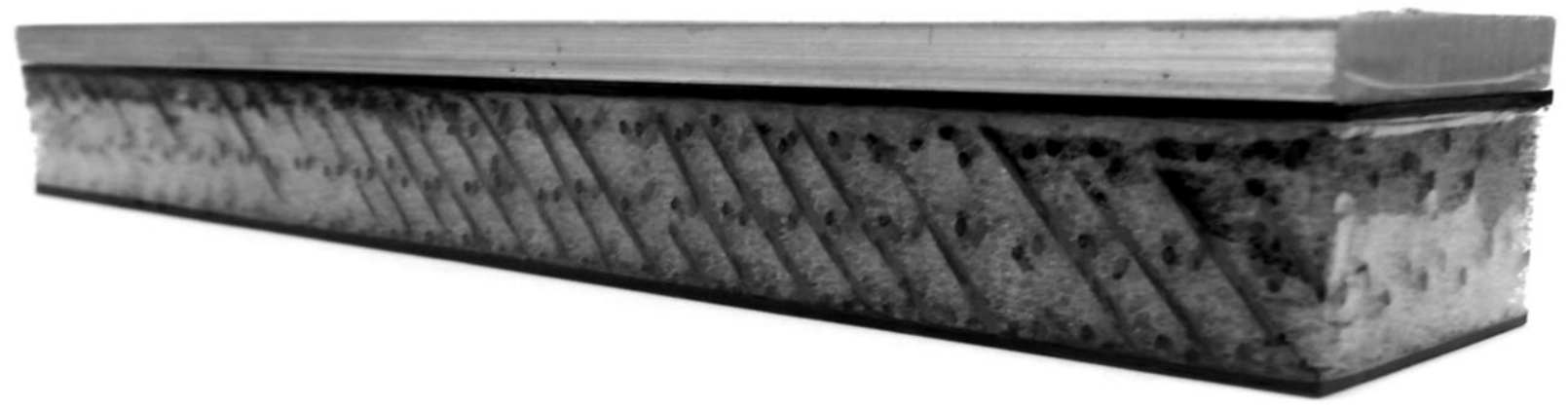

Figure 9. Fracture sample for measuring 3DFRFC properties. (not shown to scale)

During testing, additional insight into the material behavior and the interaction of the discrete constituents of the 3DFRFC will be gained through the use of the digital image correlation (DIC) capabilities of the Composite Structures Laboratory at the University of Michigan. This capability will allow for the mapping of the two dimensional strain fields on the surface of the specimen throughout the test. This capability will provide critical insight and allow for a deeper understanding of the microstructure interaction of the 3DFRFC and aid in the development of models capable of capturing this interaction.

Due to the complexity of the 3DFRFC, there are additional challenges to consider when considering test methods for determining the bulk fracture properties. The highly discretized nature of the reinforcement within the core results in a significant region of the material near the edges with partially bonded fibers. These severed reinforcements can no longer transfer load and are not representative of the bulk material. In order to determine the effective bulk properties, tests will be conducted on three specimen sizes and the effective fracture properties will be determined using two methods. The first method uses the first two test sizes and backs out the critical energy release rate of the bulk by assuming that the total can be calculated as an area weighted average of the critical energy release rate values for the partially bonded region and the fully bonded region. The third test size is used to validate this measurement. The second method bases the calculations on number of pins fully bonded for each sample to determine an effective area.

\section{Conclusion}

The experimental investigation of the Mode I and Mode II fracture of 3DFRFC sandwich composites is ongoing. The quantification of the effective bulk critical energy release rate for the 3DFRFC will be important to the modeling of 3DFRFC sandwich structures with manufacturing induced defects, damage, and core-to-facesheet delamination that can reduce the strength of sandwich composites. The outcomes of this research will provide the critical understanding and engineering tools required to fully exploit the benefits of advanced three-dimensionally reinforced sandwich structures in current and future spacecraft and launch vehicles, while having transformative impacts to the ability to utilize advanced materials in commercial aerospace and non-aerospace applications. 


\section{Acknowledgments}

The authors gratefully acknowledge The Aerospace Corporation for providing the material used for this study. This work was supported by a NASA Office of the Chief Technologist's Space Technology Research Fellowship.

\section{References}

${ }^{1}$ NidaFusion SXO/SXF, http://www.nida-core.com/spanish/fusiprod_sxosxf.htm, 2006-2008, Nida-Core Corporation.

${ }^{2}$ TYCORE@ Products, http://www.webcoreonline.com/tycor\%C2\%AE-products, 2011 WebCore Technologies.

${ }^{3}$ Ural, A., A. T. Zehnder, A. R. Ingraffea, "Fracture mechanics approach to facesheet delamination in honeycomb: measurement of energy release rate of the adhesive bond," Engineering Fracture Mechanics, Volume 70, Issue 1, January 2003, Pages 93-103, ISSN 0013-7944, 10.1016/S0013-7944(02)00024-3.

${ }^{4}$ Prasad, S., L.A. Carlsson, "Debonding and crack kinking in foam core sandwich beams-II. Experimental investigation," Eng Fract Mech, 47 (6) (1994), pp. 825-841.

${ }^{5}$ Cantwell, W.J., P. Davies, "A test technique for assessing core-skin adhesion in composite sandwich structures," J Mater Sci Lett, 13 (3) (1994), pp. 203-205.

${ }^{6}$ Cantwell, W.J., P. Davies, "A study of skin-core adhesion in glass reinforced sandwich materials," Appl Compos Mater, 3 (6) (1996), pp. 407-420.

${ }^{7}$ Cantwell, W.J., R. Scudamore, J. Ratchliffe, P. Davies, "Interfacial fracture in sandwich laminates," Compos Sci Technol, 59 (14) (1999), pp. 2079-2085.

${ }^{8}$ Carlsson, L.A., "On the design of the cracked sandwich beam (CSB) specimen," J Reinf Plastic Compos, 10 (4) (1991), pp. $434-444$

${ }^{9}$ Zenkert, D., "Strength of sandwich beams with interface debonding," Compos Struct, 17 (4) (1991), pp. 331-350.

${ }^{10}$ Zenkert, D., "PVC sandwich core materials:fracture behaviour under mode II loading and mixed-mode conditions," Mater Sci Eng, A108 (1-2) (1989), pp. 233-240.

${ }^{11}$ Zenkert, D. "Strength of sandwich beams with interfacial debondings in the core," Compos Struct, 15 (4) (1990), pp. 279299.

${ }^{12}$ Davidson, B.D., V. Sundararaman, "An unsymmetric double cantilever beam test for interfacial fracture toughness determination," Int J Solids Struct, 34 (7) (1997), pp. 799-817.

${ }^{13}$ Sundararaman, V., B.D. Davidson, "An unsymmetric end-notched flexure test for interfacial fracture toughness determination," Eng Fract Mech, 60 (3) (1998), pp. 361-377.

${ }^{14}$ Davidson, P., A. M. Waas, and C. S. Yerramalli, "Experimental determination of validated, critical interfacial modes I and II energy release rates in a composite sandwich panel," Composite Structures, Volume 94, Issue 2, January 2012, Pages 477-483, ISSN 0263-8223, 10.1016/j.compstruct.2011.08.007.

${ }^{15}$ Zenkert, D., A. Shipsha, and M. Burman, "Fatigue of Closed Cell Foams," Journal of Sandwich Structures and Materials, 8 , 2006, pp. 517-538.

${ }^{16}$ Goyal, V., J. Rome, P. Schubel, J. Tuck-Lee, G. Steckel, and D. Patel, "Strength Evaluation of Foam Core Sandwich Structures with Butt Joints," 25th Annual Technical Conference of the American Society for Composites. September 20-23, 2010, Dayton, $\mathrm{OH}$.

${ }^{17}$ FM $1300 \quad$ Epoxy $\quad$ Film Adhesive, August $2011, \quad$ Technical Datasheet, http://www.cemselectorguide.com/pdf/FM_300_081211.pdf.

${ }^{18}$ Liu, T., Z. Deng, and T. Lu, "Analytical Modeling and Finite Element Simulation of the Plastic Collapse of Sandwich Beams with Pin-reinforced Foam Cores", International Journal of Solids and Structures, 45, 2008, pp. 5127-5151.

${ }^{19}$ Alfano, G. and M. Crisfield, "Finite Interface Elements for the Delamination Analysis of Laminated Composite Structures: Mechanical and Computational Issues," Journal for Numerical Methods in Engineering, 50, 2001, pp. 1701-1736.

${ }^{20}$ Goyal, V., E. Johnson, and C. Dávila, "Irreversible Constitutive Law for Modeling the Delamination Process Using Interfacial Surface Discontinuities," Composite Structures, 65, 2004, pp. 289-305.

${ }^{21}$ Xie, D., and A. M. Waas, "Discrete cohesive zone model for mixed-mode fracture using finite element analysis," Engineering Fracture Mechanics, 73, 2006, pp. 1783-1796.

${ }^{22}$ Wang, H., and T. Vu-Khanh, "Use of end-loaded-split (ELS) test to study stable fracture behaviour of composites under mode II loading," Composite Structures, Volume 36, Issues 1-2, September-October 1996, Pages 71-79, ISSN 0263-8223.

${ }^{23}$ Kier Z.T., A.M. Waas, J. Rome, V. Goyal, P. Schubel, G. Steckel, D. Patel, and Y. Kim, "Modeling Failure of 3D Fiber Reinforced Foam Core Sandwich Structures with Defects." 53rd AIAA/ASME/ASCE/AHS/ASC Structures, Structural Dynamics and Materials Conference, Honolulu, HI (2012) 